\title{
Mode Selection in Transportation System: Implications of Quality Function Deployment
}

\author{
Najmi Kamariah \\ Business Administration Study Program STIA LAN Makassar \\ E-mail address : najmikamariah@gmail.com
}

Oktavianus Riza Ganna

Student Doctoral Degree of Hasanuddin University, Faculty of Economics

E-mail address: reza.ganna@vale.com

\author{
Abdul Rahman Kadir \\ Professor in Economic Study of Hasanuddin University \\ E-mail address: rahmankadir90@yahoo.com
}

\begin{abstract}
This article, proposing Quality Function Deployment (QFD) as a product and service design technique, can be used to make decisions. Implementation of the process is done by conceptualizing problems, collecting data, and analyzing and reporting results. In this process, the required product innovation as well as the needs of the employees, as internal organizational clients, are determined. These results are then used to design KPIs that relate to consumer perspectives as well as internal perspectives (Saha and Ahmed, 2012).

This framework is manifested in a diagram called the House of Quality (HOQ). Nevertheless, as a tool developed from a practical level, little theoretical and empirical reviews attempt to provide sufficient information to assure the benefits of QFD in the transport context. Therefore, we review the theoretical foundations that can be used to support QFD and present a number of empirical findings from the literature that can be used to evaluate the feasibility of $Q F D$ in the selection of modes of transport.

Because it is a practical tool, QFD is rarely discussed through theoretical approach. Hermann et al (2012) state that QFD has no theoretical basis at all. However, it is important to support QFD from the relevant theoretical aspects to explain how this system can work in promoting the improvement of product and service quality.

\section{Introduction}

A well-designed transport system becomes an important key to economic wellbeing for a region (Silva et al, 2015; Diamond, 2014). This is due to the movement between two points connected in the transportation system costing, and these costs are paid not to other economic agents, but lost in various forms, from fuel, air pollution to disturbances to the ecosystem, including the propagation of certain diseases. Consequently, one attempt to produce an optimal transport system is to minimize the costs incurred by a mode of transport in transit between two points in the system.

Included in the effort of the efficiency of the transport system is the determination of which mode of transportation is most appropriate to connect the two points in the system by weighing various aspects of the system, including the cost efficiency aspect. Each mode has its own advantages and disadvantages. Air transport, for example, is always in a situation on the brink of failure, with the risk of delay is very large compared to other transportation systems (Gimera et al, 2005). Renewable energy technology to
\end{abstract}


replace the fossil fuel-based technology that began to be put forward is still in the very early stages and not practical to carry large quantities of goods (Tessum et al 2015, Yaabuchi et al, 2015).

For one single criterion, the selection of modes of transportation is relatively easy. For example, nowadays smartphones have evolved applications that are able to pick the most efficient vehicles in picking up passengers and bringing them to destinations in major cities of the world (Isley et al, 2016). However, when the criteria used for consideration are numerous, only technology-based systems such as GPS cannot be used. To that end, various management tools put forward to solve this problem.

In this article, we propose that Quality Function Deployment (QFD), a product and service design technique, can be used to make transportation-relevant decisions oriented to the number of criteria determined by consumers themselves (Sahut and Kucerova, 2011). However, as a tool developed from a practical level, little theoretical and empirical reviews attempt to provide sufficient information to assure the benefits of QFD in the transport context. Here we review the theoretical foundations that can be used to support QFD and present a number of empirical findings from the literature that can be used to evaluate the feasibility of QFD in the selection of modes of transport.

\section{Theoretical Foundation of QFD}

QFD was first developed at Kobe Mitsubishi shipyard, by Yoji Akao and Shigeru Mizuno, to improve ship design and production processes, in the late 1960s (Duru et al., 2013). QFD is generally defined as "a structured framework for translating consumer requirements into appropriate technical requirements for product/service development to satisfy consumers" (Lee and Kim, 2008: 5). Implementation of the process is done by conceptualizing problems, collecting data, and analyzing and reporting results. In this process, the required product innovation as well as the needs of the employees, as internal organizational clients, are determined. These results are then used to design KPIs that relate to consumer perspectives as well as internal perspectives (Saha and Ahmed, 2012).

This framework is manifested in a diagram called the House of Quality (HOQ). Figure 1 shows an illustration of HOQ and its elements: 
Figure 1 House of Quality

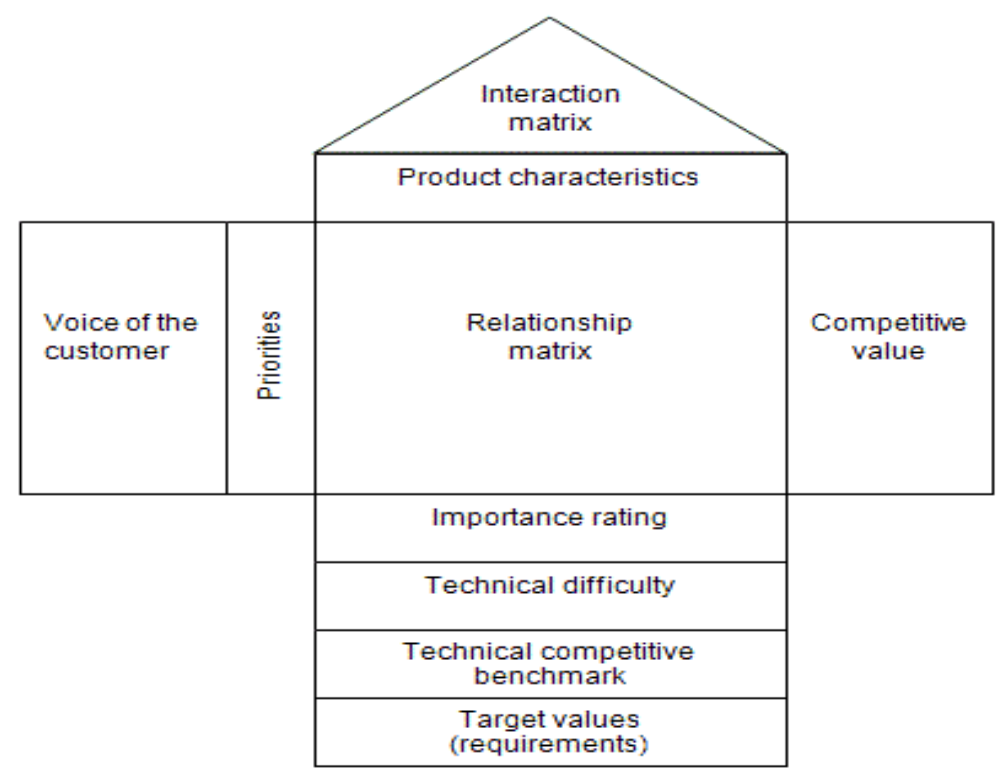

The input elements of the HoQ include the elements of the voice of the customer, the priority (the importance rating of each voice), the elements of competitive value (the company's performance rating, the competitor's performance rating, and the target value for the company against various consumer sounds), elements product characteristics in the form of technical requirements to satisfy the voice of the consumer, a relationship matrix that provides a rating of relationships between various sound combinations of consumers and technical requirements, an interaction matrix that provides correlation ratings between various combinations of technical requirements (HoQ roof), technical difficulty on various technical requirements, performance of companies and competitors in the assessment of technical competitiveness, and values target against various technical requirements (Bedi, 2006).

Based on these inputs we will obtain outputs of either absolute or relative weight values of various technical requirements useful for determining priorities in technical design, continuing to product characteristics, manufacturing/purchasing operations, and finally production/quality control, through a series of inward QFD processes.

Because it is a practical tool, it is rarely discussed about the theoretical aspects of QFD. Hermann et al (2012) state that QFD has no theoretical basis at all. However, it is important to support QFD from the relevant theoretical aspects to explain how this system can work in promoting the improvement of product and service quality. A number of theories that can be used explain the aspects of QFD include:

\section{Knowledge-Based Company Theory (Knowledge-Based View of Firm)}

Knowledge-based company theory (Grant, 1996) is one of the theories of companies that try to explain and predict the structure and behavior of the company. This theory sees that knowledge is the only sustainable resource in achieving competitive advantage (Rajib et al, 2013). Knowledge here can mean getting used to, equipped with, conscious, remembering, recollecting, recognizing, discriminating, understanding, 
interpreting, being able to explain, being able to demonstrate, able to talk, and capable of doing, which can be distinguished entirely into knowledge of and knowledge of how (Grant, 1996). This knowledge is used by companies to create value, either by means of production (converting inputs to output) or arbitration (moving products from one market to another). Knowledge is transferred using communication while knowledge of how it is transferred through the application. This transfer of knowledge depends in part on the potential knowledge for aggregation.

The company itself is an institution of knowledge application. This view is different from the previous opinion that the company is a knowledge-creating institution. Without ignoring this, Grant (1996) emphasizes that companies are essentially using knowledge and knowledge to come from within or outside the company. The application of knowledge is then executed with rules and directives, sorting, routine, and problemsolving and group decision making. Its basic capital is shared knowledge, which consists of language, other forms of symbolic communication, the introduction of the domain of individual knowledge, the komonalitas knowledge of specialization, and the shared meaning. On a broader picture, this means the company is running a process that starts from the creation of knowledge, integration of knowledge, and ends in the application of knowledge. In order for the efficient application of knowledge possible, the company must be a multiproduct company, resulting incongruence between the knowledge domain and the company's product domain.

In the view of knowledge-based enterprise theory, QFD is one tool to create knowledge by collecting data from consumers, markets, and internal. The QFD framework becomes a basic capital used to integrate knowledge from these various sources, then translate it into the application of knowledge. Therefore, QFD is a tangible form of corporate model that is described by the theory of knowledge-based company Grant (1996).

\section{Theory of the End-Tool (Means-End Theory)}

The final tool theory explains how consumers perceive a product or service more than just the functional nature of a product. More precisely, the final tool theory states that the way consumers relate to products is to form three interrelated levels of product nature (concrete and abstract), the consequences of their use (functional and psychological), and personal values (instrumental and terminal). The first consumer will perceive the attributes of the product, then relate it to the benefits of the product, and ultimately, lead to the values held by the consumer. If a product passes through these three levels of evaluation, the product will be desired, or else the product will be rejected (Leao and de Mello, 2007).

QFD connects between consumer values with the attributes and benefits that a product can provide (Herrmann et al, 2012). The task of QFD is to match the value of consumers with the attributes of their products so as to produce the benefits that attribute attributes to that value. Consumer value is represented by the voice of consumer while product attributes are represented by product technical requirements. In this way, QFD works in line with the final-tool theory in producing the final design that can be bought or accepted by consumers.

\section{Stakeholder Management Theory}


Stakeholder management theory argues that the goal of the company is to coordinate the interests of stakeholders (Donaldson and Preston, 1995: 79). Failure to coordinate the interests of stakeholders will lead to a failure of company performance or even company closure. While anyone who becomes a stakeholder of the company becomes a contentious area, it is acceptable that both consumers and employees are important stakeholders of the company, each representing external stakeholders and internal stakeholders. The interests of both are facilitated by QFD because the perspective of the consumer, as well as the perspective of the employees, are included in the framework.

Furthermore, stakeholder management theory states that the task of management to consumers is how to keep consumers from taking on what the market offers them, but have expectations of what they need to receive, and the company will provide this (Verbeke and Tung, 2013 ). By cultivating this awareness of expectation, the company can be more prominent than its competitors, and in turn get loyal customers. QFD captures what consumers expect and provides what consumers expect.

On the other hand, stakeholder management theory also states that management's job to employees is how to keep employees from soliciting incentives from each of their efforts against the company. Employees endeavor to experience desensitization, that is to experience the reduction of emotional-based physiological reactivity to the stimuli of the enterprise (Verbeke and Tung, 2013). Attempts to desensitize employees are to provide a minimum-maintained minimum quality framework for retaining employees. One form of desensitization efforts is to constantly listen to input from employees. This is facilitated by QFD by providing space for employees stating the technical capabilities of products they know to meet consumer values.

In turn, QFD can be viewed as one form of stakeholder management. While not all stakeholders are facilitated in the QFD, it has enough to address two core stakeholders, and broader stakeholder management theory can link QFD with other stakeholders such as competitors, suppliers, and governments.

\section{Theory of Quality Management}

Just like QFD, quality management is a concept that is lifted from the practical, rather than theoretical, realm. Quality management evolves primarily based on direct experience in industry rather than careful consideration of conceptual analysis and empirical testing. Indeed, quality management has long developed and begins to get a clearer theoretical basis. This is achieved by looking at the common elements of the various types of quality management approaches practitioners put forward in the field. Scholars conclude that there are three main components, namely consumer focus, continuous improvement, and total engagement (Prajogo and McDermott, 2005). Correspondingly, the quality management theory argues that the company's total commitment to the total quality setting allows leaders to manage and harmonize human activities within the enterprise to achieve common goals (Sadikoglu and Zehir, 2010). More broadly, by involving other core elements, the quality management theory means that total commitment is directed to meeting consumer needs (consumer focus) and running it sustainably.

QFD takes the appropriate steps with the above quality management theory formulation. QFD cultivates all design activities in a single framework, making the 
product design a totality. This totality is focused on the needs of consumers, termed voice of the consumer. Only sustainable components are outside the QFD because it depends on leadership to choose whether to repeat the QFD procedure continuously on subsequent occasions or choose to use it only once.

$\mathrm{Zu}$ et al (2008) integrate a broader conception of quality management into two areas, namely infrastructure and core. Infrastructure includes customer relationships, supplier relationships, and labor management, while core components include quality information, product/service design, and process management. The description of $\mathrm{Zu}$ et al (2008) is more pertinent to QFD because customer relationships are hypothesized to have an effect on providing quality information, which in turn provides product/service design that will lead to quality performance and ultimately business performance. Moreover, quality information is not only derived from consumer relations but also from labor management. These two sources have been facilitated directly by QFD. In fact, Zu et al (2008) state that labor-management continues to be in touch with product/service design, which also gets input from quality information. Again, QFD facilitates this because the design process is indeed run by the workforce based on the quality information provided by consumers through QFD.

\section{Empirical support for QFD}

Several claims have been made to demonstrate the effectiveness of QFD in field applications. Many practitioners rate that QFD can reduce their product development and service development cycle time by up to $75 \%$ with the same value of customer satisfaction (ASQ, 2016). Implementation at Toyota compares the pre-production cost and startup of Toyota Auto Body in 1977 before QFD and 1984 when it has implemented QFD. They claimed that QFD was able to reduce costs by more than 60\% (Hauser and Clausing, 1988). Meanwhile, a comparison of the number of design changes between Japanese and US automakers shows that design changes continue to occur after the first automobiles are in production in the United States, while in Japan, design changes have ceased before the first car was produced (Hauser and Clausing, 1988). For now, companies like Samsung use QFD as their design tool (Lager, 2016).

Truly, a more careful investigation yields a wide variety of results. Carnevalli and Miguel (2007) analyzed 157 QFD-related articles showing that only 20\% of articles suggesting benefits are likely to increase reliability, reduction in project changes, decreasing design time, decreasing design costs, rising incomes, and decreasing claims. Meanwhile, $80 \%$ more expressed on invisible benefits such as flexibility, improved communication, helped decision-making and priorities, created cross-functional working groups, improved and sustained corporate knowledge, and increased consumer satisfaction. Cameron and Quinn (2006: 49) are pessimistic at QFD, seeing that QFD will not always work, as with other quality management interventions, if not considering changes in organizational culture. In the theory of organizational culture-based network of competitive value (Competing Value Network), QFD can only touch the culture of market-based organizations, not clan-based, adocracy, or hierarchy. In order for companies with these three dominant cultures to successfully run QFD, there must be an attempt to change organizational culture in the direction of market culture and this will take a long time due to the nature of the organizational culture that is difficult to change. 
Another study conducted by Lager (2005) only reviewed nine studies at the industry level. The Lager (2005) study states that the claim that QFD may shorten product time to reach the market has no scientific support at all. According to Punchihewa and Gyi (2015), this suggests that processes using natural structures such as QFD will take time to deliver reliable results. Truly, Lager (2005) supports that QFD is indeed capable of delivering better products and improving the quality of dissemination and retrieval of information for the needs of design decision making. Therefore, Lager (2016) and Tottie et al (2016) continue to use the QFD methodology to develop new products, including within the B2B (Business to Business) framework.

\section{Implementation of QFD}

More contemporary studies continue to apply QFD in various contexts. Wang et al (2010) use QFD to transform organizational performance measures into project performance measures. In this way, they are able to develop a risk management framework, which in turn can be used by $\mathrm{R} \& \mathrm{D}$ projects to focus on achieving corporate objectives. This research is one example of how QFD is used in the field of corporate $\mathrm{R}$ $\&$ D. Another area that started to be touched by QFD is the development of the competitiveness of SMEs (Massa and Gessa, 2016) and the product of fast food restaurants (Baran and Yildiz, 2015).

In different directions, many researchers have begun to apply the theory of the fading set in an attempt to overcome the qualitative nature of the product development process. This trend began to be observed by Carnevalli and Miguel (2007). Chen and Ko (2009) for example, uses fuzzy QFD QFD as an alternative to discrete, by combining them with FMEA (Failure Modes and Effects Analysis). Meanwhile, OuYang and Weng (2011) combine QFD with TRIZ to better guarantee that the product is completely new. TRIZ (Teoriya Resheniya Izobretatelskikh Zadatch - Inventive Problem Solving Theory) is a comprehensive patent analysis approach.

\section{Mode Selection in Transportation System}

The problem of selecting modes in the transportation system is done by various approaches. Generally, the approach used is the optimization approach based on the mathematical model of the linear program with a number of pre-determined parameters and is quantitative (eg Kutanoglu and Lohiya, 2008 and Celikbilek and Suer, 2013).

Linear program based mode selection systems have drawbacks. This weakness lies in the difficulty of determining the values relevant to the constraint coefficients in a linear program. In addition, the determination of certain objective functions for linear programs is a complex issue. Moreover, even if the objective function is known, the linear model only considers a single purpose function, whereas in reality, not only the function of the objectives of each organization can vary, but also one organization or program can have a number of objective functions that must be met. Finally, in fact, not all variables in the model can be quantified linearly, and if they can be quantified linearly, their value may vary over time. For that, it takes a simpler mode selection method, handling a number of purposes (consumer value) at once, the value of the goal can be quantified clearly using discrete or fuzzy systems, and does not require linearity assumptions. This can be fulfilled by QFD based mode selection. 


\section{QFD Implications for Transportation Mode Selection}

The use of QFD in the field of transportation is directed to the design of transportation equipment to the intermodal problem. Melemez et al (2013) use QFD to design trailers to transport wood from the forest. Wolfsmayr and Rauch (2014), for example, use QFD to assess the constraints and support factors for capital turnover from truck to rail in sending wood from the forest. QFD is chosen over the SWOT approach because it is more technical and conscientious than SWOT, although admittedly more formal and time-consuming.

Use of moda selection, however, has not been found. This opens up opportunities for QFD implications in the selection of modes of transportation. The practical application of moda selection is to determine the criteria of the desired consumer of the mode for transporting a product. This criterion reflects the consumer value required. Furthermore, the technical specifications of each mode of transportation are adjusted to meet the criteria that consumers expect. For more details, Figure 2 shows an example of HOQ villageing with the purpose of selection of modes of transport.

Figure 2 HOQ Selection Mode of Transportation

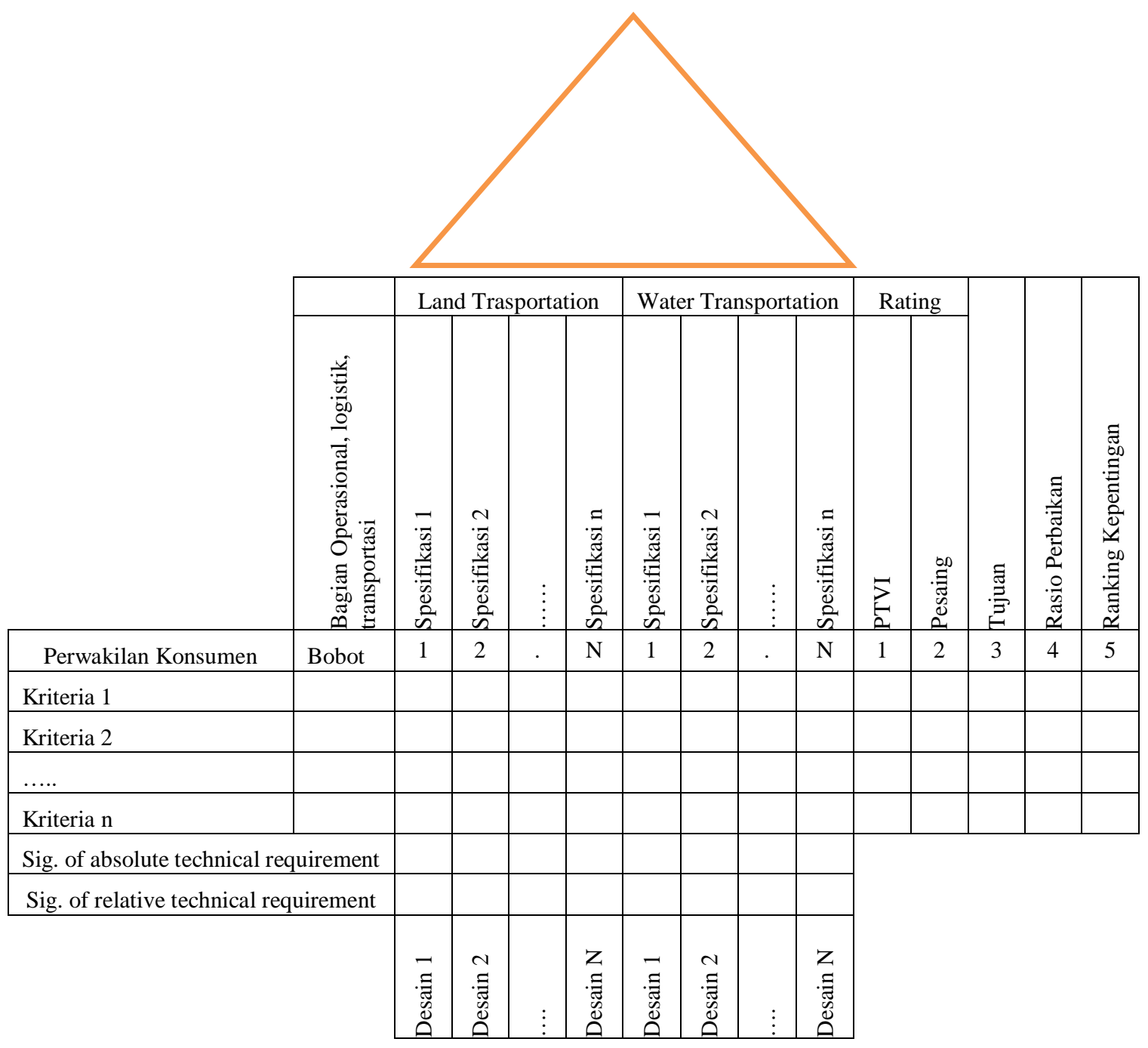


For maximum results, QFD needs to be combined with other methods such as AHP and SWOT. Combination methods are commonly used for the purpose of choosing something. Rajesh and Malliga (2013) use AHP and QFD to strategically select suppliers,

For product criteria interviews, representatives are asked to provide an opinion on what value they want from the company's product. Interviews are open and the researcher's job is to get as many product criteria as they want, without first assessing whether the criteria can be improved through logistics solutions or not. They are also asked whether they have other suppliers other than the company or have other suppliers other than the company. This information is necessary for market comparison data.

For the internal company, interview/discussion of the technical specification is done by considering the voice of consumer and technical specification only directed to two groups, that is a group of land transportation and water transportation group. That is, there are two dimensions of the technical specification to be assessed.

At the SWOT stage, the SWOT table is arranged and pursed into two alternatives between ground transportation and water transport. The FGD will produce which of the two types of modes to be selected based on the proposed technical design for the repair or total weight of the technical specifications. The FGD also discussed other issues worthy of consideration before decision making. The chosen mode will be prioritized to be developed to meet consumer criteria. Meanwhile, unselected modes will be left like the current initial conditions.

\section{Conclusion}

In this article, we are trying to expand the application and benefits of QFD in the field of transportation mode selection. QFD can assist in providing the ability in moda selection because it is multi-criteria, simple, and does not require linearity assumptions. The combination with AHP allows the selection criteria to be more precisely weighted while the SWOT application at the end of the process is able to push decisions between two alternatives based on the considerations generated from QFD. Meanwhile, AHP with discrete value is criticized for raising the bias of the evaluator for weighing linguistic decisions with a single value. The solution to this problem is the application of vague set theory in the AHP evaluation. Consequently, the whole theoretical framework follows the AHP sequence $\diamond$ QFD $\diamond$ SWOT.

The selection of the QFD-based mode meets the theoretical needs associated with knowledge-based enterprise theory. With QFD, companies are able to decide which mode of transportation is the most knowledge-based that is acquired directly from consumers and internally. The use of QFD in moda selection also ensures that consumers get the most optimum benefit based on the values they are prioritizing, in accordance with the final tool theory. For the company, basing the selection of modes of transportation on the consumer and technical specifications is also the application of stakeholder management that makes consumers have higher expectations and employees can feel their opinions are heard in the mode selection. Finally, QFD in moda selection is also the application of quality management in moda selection that reflects the total involvement of the company in an effort to provide the best service for the consumer in a sustainable manner.

Ultimately, the application of QFD in modes selection can provide practical benefits for remote mining companies faced with the mode of transportation mode dilemma. Some firms are faced with a choice problem between land, sea and air modes, 
each of which has advantages and disadvantages to deliver products directly to consumers. By submitting the value or selection criteria to the consumer and adjusting to company specifications, it will be obtained certainty about which modes can be developed to optimize the company's transportation system they have.

\section{Reference}

ASQ (American Society for Quality) (2016) What is Quality Function Deployment (QFD)?http://asq.org/learn-about-quality/qfd-quality-functiondeployment/overview/overview.html

Baran, Z., \& Y1ld1z, M. S. (2015) Quality Function Deployment and Application on a Fast Food Restaurant. International Journal of Business and Social Science Vol. 6, No. 9; 122-131

Bedi, K. (2006). Automating the quality function deployment house of quality. U21 Global Working Paper

Cameron, K.S., Quinn, R.E (2006) Diagnosing and Changing Organizational Culture. San Francisco: Jossey-Bass

Carnevalli, J. A., \& Miguel, P. A. C. (2007). Literature review, classification, and analysis on QFD: types of research, difficulties of the use, and benefits of the method. Gestão \& Produção, 14(3), 557-579.

Çelikbilek, C., \& Süer, G. (2013). Joint Optimization Model for Manufacturing Scheduling and Transportation Mode Selection. In Asian Conference on Management Science and Applications (ACMSA 2013), Kunming-Yunnan, China.

Chen, L. H., \& Ko, W. C. (2009). Fuzzy approaches to quality function deployment for new product design. Fuzzy sets and systems, 160(18), 2620-2639.

Diamond, J. (2014). Reversals of national fortune, and social science methodologies. Proceedings of the National Academy of Sciences, 111(50), 17709-17714.

Donaldson, T., \& Preston, L. E. (1995). The stakeholder theory of the corporation: Concepts, evidence, and implications. Academy of Management Review, 20(1), 65-91.

Duru, O., Bulut, E., Huang, S. T., \& Yoshida, S. (2013). Shipping Performance Assessment and the Role of Key Performance Indicators (KPIs):'Quality Function Deployment'for Transforming Shipowner's Expectation. Available at SSRN 2195984.

Grant, R. M. (1996). Toward a knowledge-based theory of the firm. Strategic management journal, 17(S2), 109-122.

Guimera, R., Mossa, S., Turtschi, A., \& Amaral, L. N. (2005). The worldwide air transportation network: Anomalous centrality, community structure, and cities' global roles. Proceedings of the National Academy of Sciences,102(22), 77947799.

Hauser, J. R., \& Clausing, D. (1988). The house of quality. Harvard business review, 66(3). 
Herrmann, A., Huber, F., Algesheime, R., \& Tomczak, T. (2006). An empirical study of quality function deployment on company performance. International Journal of Quality \& Reliability Management, 23(4), 345-366.

Isley, S. C., Stern, P. C., Carmichael, S. P., Joseph, K. M., \& Arent, D. J. (2016). Online purchasing creates opportunities to lower the life cycle carbon footprints of consumer products. Proceedings of the National Academy of Sciences, 113(35), 9780-9785.

Kutanoglu, E., \& Lohiya, D. (2008). Integrated inventory and transportation mode selection: A service parts logistics system. Transportation Research Part E: Logistics and Transportation Review, 44(5), 665-683.

Lager, T. (2005). The industrial usability of quality function deployment: a literature review and synthesis on a meta-level. $R \& D$ Management, 35(4), 409-426.

Lager, T. (2016). Managing Innovation \& Technology in the Process Industries: Current Practices and Future Perspectives. Procedia Engineering, 138, 459-471.

Leão, A. L. M., \& Mello, S. C. (2007). The means-end approach to understanding customer values of an online newspaper. BAR-Brazilian Administration Review, 4(1), 1-20.

Lee, C., \& Kim, S. H. (2008). A Method to Reflect Corporate Business Strategy on Information System Assessment Using the Quality Function Deployment and Analytic Hierarchy Process.

Massa, G., \& Gessa, N. (2016). QFD for a SME Network of the Wood Sector to Improve Competitiveness and Sustainability. In Sustainable Design and Manufacturing 2016 (pp. 501-511). Springer International Publishing.

Melemez, K., Di Gironimo, G., Esposito, G., \& Lanzotti, A. (2013). Concept design in the virtual reality of a forestry trailer using a QFD-TRIZ based approach. Turkish Journal of Agriculture and Forestry, 37(6), 789-801.

OuYang, K., \& Weng, C. S. (2011). A new comprehensive patent analysis approach for new product design in mechanical engineering. Technological Forecasting and Social Change, 78(7), 1183-1199.

Prajogo, D. I., \& McDermott, C. M. (2005). The relationship between total quality management practices and organizational culture. International Journal of Operations \& Production Management, 25(11), 1101-1122.

Punchihewa, H. K., \& Gyi, D. E. (2015). Reducing work-related Musculoskeletal Disorders (MSDs) through design: Views of ergonomics and design practitioners. Work, 53(1), 127-142.

Rajesh, G., \& Malliga, P. (2013). Supplier selection based on AHP QFD methodology. Procedia Engineering, 64, 1283-1292.

Rajib, M., Uddin, S., Alam, M., \& Arefin, M. (2010). A Case Study on Integrating Logistics Information Systems with the Corporate Resources. InProceedings of 7th International Conference on Innovation and Management, Wuhan, China (pp. 1595-1600).

Sadikoglu, E., \& Zehir, C. (2010). Investigating the effects of innovation and employee performance on the relationship between total quality management practices and firm performance: An empirical study of Turkish firms.International Journal of Production Economics, 127(1), 13-26. 
Saha, A. K., \& Ahmed, R. (2010). Formulation of the Strategy for Constructing a Balanced Scorecard (Academic Scorecard) for a University.Dhaka University Journal of Management, 2(1), 69-84.

Sahut, J. M., \& Kucerova, Z. (2011). Quality Management of Internet Banking Services. Available at SSRN 1755497

Silva, R., Kang, S. M., \& Airoldi, E. M. (2015). Predicting traffic volumes and estimating the effects of shocks in massive transportation systems.Proceedings of the National Academy of Sciences, 112(18), 5643-5648.

Tessum, C. W., Hill, J. D., \& Marshall, J. D. (2015). Reply to Oron: Electric vehicles provide an opportunity to reduce environmental health effects of transportation. Proceedings of the National Academy of Sciences of the United States of America, 112(30), E3974.

Tottie, M., Lager, T., \& Nordqvist, S. (2016). From customer understanding to product under-standing: Collaboration with industrial lead users in a B2B context. Journal of Business Chemistry, 13(2), 64.

Verbeke, A., \& Tung, V. (2013). The future of stakeholder management theory: A temporal perspective. Journal of Business Ethics, 112(3), 529-543.

Wang, J., Lin, W., \& Huang, Y. H. (2010). A performance-oriented risk management framework for innovative R\&D projects. Technovation, 30(11), 601-611.

Wolfsmayr, U. J., \& Rauch, P. (2014). Primary forest fuel supply chain: assessing barriers and drivers for the modal shift from truck to train. Silva Fennica, 48(5).

Yabuuchi, N., Takeuchi, M., Nakayama, M., Shiiba, H., Ogawa, M., Nakayama, K., ... \& Sato, K. (2015). High-capacity electrode materials for rechargeable lithium batteries: aLi3NbO4-based system with cation-disordered rocksalt structure. Proceedings of the National Academy of Sciences,112(25), 7650-7655.

Zu, X., Fredendall, L. D., \& Douglas, T. J. (2008). The evolving theory of quality management: the role of Six Sigma. Journal of Operations Management, 26(5), 630-650. 